\title{
Covid-19 and endocrinology - intersections: a review of current knowledge on physiopathological associations
}

\begin{abstract}
Introduction: The coronavirus-19 pandemic has reached historic dissemination, with a huge impact on physical, social and psychological well-being; understanding the pathophysiology of the disease is extremely important, as it can lead to effective therapy formulations.
\end{abstract}

Objective: In this review, we will explore the current evidence surrounding the interactions of endocrine conditions and possible treatment targets.

Methods: we reviewed the Medline, EMBASE and LiLACS databases using the keywords "COVID-19" or "SARS-CoV-2", crossing them with the keywords "Obesity", "Diabetes Mellitus", "Vitamin D”, “ Estradiol ", Testosterone ", " Male "and" Female ”; we will describe the results found in a narrative way.

Results: The association of diabetes mellitus, obesity and worse prognosis of people infected with the SARS-CoV-2 virus, as well as the gender dimorphism of severity and the role of vitamin $\mathrm{D}$ deficiency will all be described.

Conclusion: There is a riddle involving SARS-CoV-2 infection, intrinsic risk factors of the host, as well as the immune response to the infection, which is still in the solution phase; a better understanding of such mechanisms can lead to the development of highly effective treatment, as well as policies of social isolation.

Keywords: endocrinology, COVID-19, obesity, Ddiabetes mellitus, vitamin D
Volume 8 Issue 4 - 2020

\begin{abstract}
Lucas Ribeiro dos Santos,' Ricardo Baer Filho, ${ }^{2}$ Thais Amanda Frank de Almeida Alves, ${ }^{2}$ Matheus Budahazi Jardine, ${ }^{2}$ Márcio Luís Duarte ${ }^{3}$

'Professor of endocrinology and physiology at Santos Medical Sciences Faculty of Lusiada University, Master of Sciences in the Evidence-Based Medicine Program UNIFESP-EPM, Brazil ${ }^{2}$ Santos Faculty of Medical Sciences - Lusiada University, Brazil ${ }^{3}$ Radiologist at Webimagem, Master of Sciences in the EvidenceBased Medicine Program UNIFESP-EPM, Brazil
\end{abstract}

Correspondence: Lucas Ribeiro dos Santos, Medical SciencesFacultyof Santos - Santos, São Paulo, Brazil, Oswaldo Cruz Street, 179 - Boqueirão, Santos - SP, I I045- I0I, Tel (I3) 3202-4500, Email Irs.ndocrino@gmail.com

Received: August 12, 2020 | Published: August 25, 2020

\section{Introduction}

Most people infected by the severe acute respiratory syndrome coronavirus 2 infection (SARS-CoV-2) present oligosymptomatic forms of disease (COVID-19). ${ }^{1}$ However, since the start of the coronavirus-19 pandemic, a few risk factors have indicated select groups with higher probability of developing more severe presentation. Obesity, diabetes mellitus and cardiovascular disease emerged as the first recognizable risk factors and predictors of mortality; ${ }^{2-3}$ afterwards, it became noticeable that male patients infected with the new SARSCoV-2 had higher odds of presenting moderate to critical forms of disease. ${ }^{4}$ Understanding the mechanisms underlying such dimorphism could help create protocols of clinical support and selection of higher risk patients that could benefit from social isolation policies, as well as reveal new targets of possible pharmacological interventions.

\section{Objectives}

In this review, we will depict the pathophysiological associations between the severity of COVID-19 and known endocrinological situations associated with worst prognosis.

\section{Material and methods}

We performed a perspective review using the key words "COVID-19" or "SARS-CoV-2", crossing them with the keywords "Obesity", "Diabetes Mellitus", "Vitamin D", "Estradiol", "Testosterone", "Male" and "Female" on the Medline, LiLACS and Embase databases from November first, 2019, to July 31 2020. Grey literature was also analyzed through the OpenGrey, MedRvx and SSRN databases. Results and implications were depicted in a narrative form. We did not place any language restrictions.

\section{Results}

\section{Obesity}

Evidence emerges that obesity-related conditions appear to worsen the effects of the virus. Chinese cohort studies of patients with COVID-19 have identified several risk factors for severe COVID-19, including age, cardiomyopathy and obesity-related complications, such as type 2 diabetes and hypertension. ${ }^{5}$ New York City data suggest that obesity, defined by a BMI of at least $30 \mathrm{~kg} / \mathrm{m}^{2}$, may be a risk factor for intensive care unit (ICU) admission among patients with COVID-19, increasing the risk up to 2-fold, especially among those under 60 years of age. ${ }^{3}$ Moreover, data from the University Hospital of Lyon, of 340 patients admitted to ICU with COVID-19 revealed that $85(25 \%)$ with severe COVID-19 had obesity, compared to $15.3 \%$ in the general French adult population. After multivariate logistic regression adjusted for age and sex, the prevalence of obesity remained higher in patients with critical COVID-19 than in those with non-critical COVID-19 (OR 1.96 [95\% CI 1.13-3.42]; $\mathrm{p}=0.018$ ). ${ }^{6}$ Likewise,in Lyon, obesity prevalence in critical COVID-19 patients in the Lille University Hospital population was 2.88 times $(95 \%$ CI 2.19-3.66) higher than in the general French population after standardization in age and sex $(p<0.0001){ }^{6}$ Underlying mechanisms remain unclear, but main hypothesis rely on restriction of pulmonary ventilation, inflammatory activity that amplifies cytokine storm, and endothelial dysfunction associated with a pro-thrombotic state that may cause microvascular thrombosis. 


\section{Pulmonary restriction}

A recent systematic review demonstrate that obese individuals presented with a reduction in lung volume and capacity as compared to healthy individuals. Reduction of total lung capacity and reduction of forced vital capacity, accompanied by reduction of the forced expiratory volume after one second was also observed. ${ }^{7}$ This restriction itself could partially explain the level of difficulty to provide adequate ventilation, and the increased need for ventilatory support, invasive or not. This is most probably due to fat tissue surrounding the upper airways, as well as the chest walls, hampering ventilatory muscles work.

\section{Inflammatory activity and thrombosis}

Coronavirus infections can trigger venous thromboembolism due to endothelial dysfunction, systemic inflammation and a procoagulatory state. ${ }^{8}$ In the autopsy of 12 patients who died of COVID-19, 58\% of the cases had deep venous thrombosis and one third of the patients had pulmonary embolism as the direct cause of death. ${ }^{9}$ It is reported in the literature that patients with COVID-19 have thrombocytopenia and elevated D-dimer and that this finding is even more frequent in patients with the severe form of the disease. ${ }^{8}$ These data suggest, then, a greater risk of disseminated intravascular coagulation (DIVC).$^{10}$ These laboratory changes may be explained by the inflammatory response caused by viral infections that promote excessive activation of the coagulation cascade and platelets. In this scenario, platelets interact with pathogen pattern recognition receptors or immunoglobulin $\mathrm{Fc}$ receptors and complement receptors promoting activation and interactions between cells that act in procoagulation. ${ }^{10}$ Pre-existing comorbidities can impair the immune response or amplify the pro-inflammatory response in patients infected with Coronavirus disease 2019 (COVID-19). Individuals with obesity have low-grade chronic systemic inflammation ${ }^{11}$ with higher activity of nuclear transcription factor kappa B and a higher ribonucleic acid expression of proinflammatory cytokines. As the innate immune response in these patients is altered, the first line of defense is characterized by a greater inflammatory response and an abnormal $\mathrm{T}$ cell response, ${ }^{12}$ exhibiting a preference for the cytotoxic $\mathrm{T}$ cell response, as well as shift towards a M1 macrophage response, amplifying viral proinflammatory response. In fact, obese individuals show an increase in interleukin- 6 and a positive correlation between C-reactive protein $(\mathrm{CRP})(\mathrm{p}=0.014)$ and waist-hip ratio $(\mathrm{WHR})(\mathrm{p}=$ $0.031) .{ }^{11,13}$

\section{Diabetes mellitus impact on severity and fatality}

As one of the most important public health problems in the $21 \mathrm{st}$ century, diabetes mellitus (DM) affects more than 400 million adults around the world. ${ }^{14}$ Evidences indicate that diabetic patients with acute viral respiratory infections usually present serious complications, such as pneumonia, acute respiratory distress syndrome, organ failure, and even death. ${ }^{15}$ In fact, the effects of DM on the severity and fatality of Covid-19 infection have gained focus on patient risk stratification.

A Meta-analysis of the association between DM and COVID-19 severity based on twelve previously published studies, with a combined total number of patients of 2455, where 232 were diabetic and 2223 were not, presented respectively 94 and 471 of severe COVID-19.
Pooled results show an over 2 times higher risk of severe cases in diabetic patients (pooled OR=2.58, $95 \%$ CI: 1.93-3.45). Furthermore, subgroup analysis results revealed a pooled OR of 3.04 (95\% CI: 2.03-4.56) for patients aged $<50$ years and 2.58 (95\% CI: $1.93-3.26)$ for patients aged $\geq 50$ years, respectively. ${ }^{2}$

The same authors evaluated the association between diabetes mellitus and COVID-19 fatality based on six published clinical studies. The total number of patients included in this analysis was 2116, in which 62 and 89 deaths from COVID-19, respectively, derived from diabetic (322 cases) and non-diabetic patients (1794 cases), granting higher risk of fatality with a pooled OR $=2.95$ (95 \% CI: 1.93-4.53). ${ }^{2}$ Another meta-analysis that included 6452 patients from 30 studies showed that DM was associated with a poor outcome compound (including mortality, severe COVID-19, acute respiratory distress syndrome [ARDS], need for ICU care),with a relative risk of $2.38(1.88-3.03, p<0.001)$. The mortality RR was of $2.12(1.44-$ $3.11, \mathrm{p}<0.001)$, and of severe COVID-19 was $2.45(1.79-3.35, \mathrm{p}<$ $0.001){ }^{16}$

Gathered data demonstrate the increased vulnerability of DM patients regarding severity and fatality by Covid-19. Diabetics presentpoor adaptive immunity, delayed T-Helper-1 type hypersensitive reactions and reduced lymphocyte proliferation. ${ }^{17}$ Defects of the innate response in diabetes results in dysfunction of granulocytes, dendritic cells, natural killer (NK), macrophages, cells, B cells, T cells and cytokine signaling. ${ }^{18}$ Interleukin-6 (IL-6), fibrinogen and C-reactive protein were reportedto be significantly higher in the patients with diabetes and COVID-19 infection. ${ }^{19}$ Mechanisms thought to be responsible for the inflammatory state in DM, as well as obesity, include activation of the nuclear factor-B (NF-B) and JUN N-terminal kinase (JNK) pathways, activation of interleukin-1 (IL-1), IL-6, TNF and recruitment and activation of immune cells. ${ }^{20}$ Such hyperactive pro-inflammatory response could enhance cytokine storm deflagrated by SARS-CoV-2 infection, as two inflammatory diseases feedback each other. ${ }^{21}$ Recently, evidence rises that metformin could attenuate this hyper-inflammatory condition, as it decreases TNF $\alpha$ and IL-6, increases IL-10 level, as well as increases activation of AMP-activated protein kinase (AMPK), which has important effects downstream in Covid-19.22 Its use has been reported to be significantly associated with decreased mortality in women in logistic regression OR $0.792(0.640-0.979){ }^{23,24}$

\section{Glycemic control and CoViD-I 9 outcomes}

Once we have stablished DM impact on COVID-19 prognosis, it is important to understand the impact of glycemic control on the mortality of diabetic patients. A retrospective multicenter study, conducted in the Hubei province, China, compared severity and mortality in patients with COVID-19 with pre-existing DM and without DM, in 7336 patients with COVID-19, being 810 diabetic. The mortality rate was significantly higher in patients with pre-existing DM compared to non-diabetic individuals $(7.8 \%$ versus $2.7 \%, \mathrm{p}<0.001$; adjusted risk ratio of 1.49). ${ }^{25}$ Furthermore, after a 28 -day follow-up, 528patients with pre-existing DM2 were stratified as poorly controlled diabetes (blood glucose $[\mathrm{BG}]>180 \mathrm{mg} / \mathrm{dl}$, median $\mathrm{BG}=216 \mathrm{mg} / \mathrm{dL}$ and median $\mathrm{HbA} 1 \mathrm{C}=8.1 \%$ ), and 282 patients as having well-controlled diabetes (BG 70-180mg/dl, median BG=116mg/dL and median $\mathrm{HbA} 1 \mathrm{C}=7.3 \%$ ). Although both groups had similar baseline age, body mass index and chest computed tomography scan at admission, a lower incidence 
of lymphopenia, lower rates of increased leukocyte and neutrophil counts and an increase in serum CRP and procalcitonin were observed in the group with Controlled BG.There was also a significantly lower frequency of pharmacological therapy in the well-controlled BG group compared to the poorly controlled group. ${ }^{25}$

In this cohort, hospital mortality rate and all-cause mortality were significantly lower in the well-controlled group compared to the poorly controlled group; after propensity analysis adjustment for multiple confounding factors, the well-controlled BG group was still considered a lower risk for all causes of mortality (adjusted hazard ratio, $0.14 ; 95 \% \mathrm{CI}, 0.03-0.60 ; \mathrm{p}=0.008) .{ }^{25}$ Moreover, a retrospective multicenter study conducted in 88 American hospitals compared a pooled group of 451 patients with diabetes (HbA1C $\geq 6.5 \%$ and / or $\geq 2 \mathrm{BG}$ measurements over $180 \mathrm{mg}$ ), in which 257 had uncontrolled glycaemia (BG $>180 \mathrm{mg} / \mathrm{dL})$. Hospitalization days (603 [SD 42.9] X 804 [SD 57.1]), and mortality (13 [14.8\%] X 40 [41.7\%]) were significantly higher amongst uncontrolled glycaemia group. ${ }^{26}$ Underlying mechanism remains unclear, but many theories have emerged. Non-enzymatic glycation of the angiotensin-converting enzyme 2 (ACE2) is exacerbated, facilitating viral infection; inflammatory, as well as pro-thrombotic response, are hyper-activated, amplifying viral tissue damage. In addition, endothelial damage, and oxygen-reactive species might play a role in this interaction of hyperglycemia and SARS-CoV-2 infection. ${ }^{27}$

\section{Dipeptidyl peptidase 4 action on viral cell infection}

The dipeptidyl peptidase 4 (DPP4) is an enzyme responsible for the inactivation of the incretin hormones released by the gut endocrine cells. It has been demonstrated that its activity is enhanced in conditions such as obesity, type 2 diabetes mellitus, and aging, ${ }^{28}$ and the use of antidiabetic drugs that inhibit this enzyme has been shown to decrease levels of IL-6, TNF-alpha. ${ }^{29}$ Moreover, in the Middle-east Respiratory Syndrome (MERS) pandemic, also caused by a coronavirus, the DPP4 was shown to facilitate interaction of the virus spike protein with the angiotensin converting enzyme (ACE) 2 , permitting cell infection. Thus, interaction of current SARS-CoV-2 with DPP4 has been postulated, as, naturally, ACE2 is little expressed in the airway tissue..$^{30}$

A recent case-control study evaluated if previous use of DPP4 inhibitors by diabetic patients had an impact on CoViD-19 infection, but failed to find this association, as use of this class of drugs was similar between diabetic patients with SARS-CoV-2 infection and with other pneumonia etiologies. ${ }^{31}$ However, there are registries of clinical trials to evaluate the use of such drugs in this context. To summarize, possible mechanisms that could explain interaction between obesity, diabetes mellitus and CoViD-19 severity are characterized in Figure 1 .

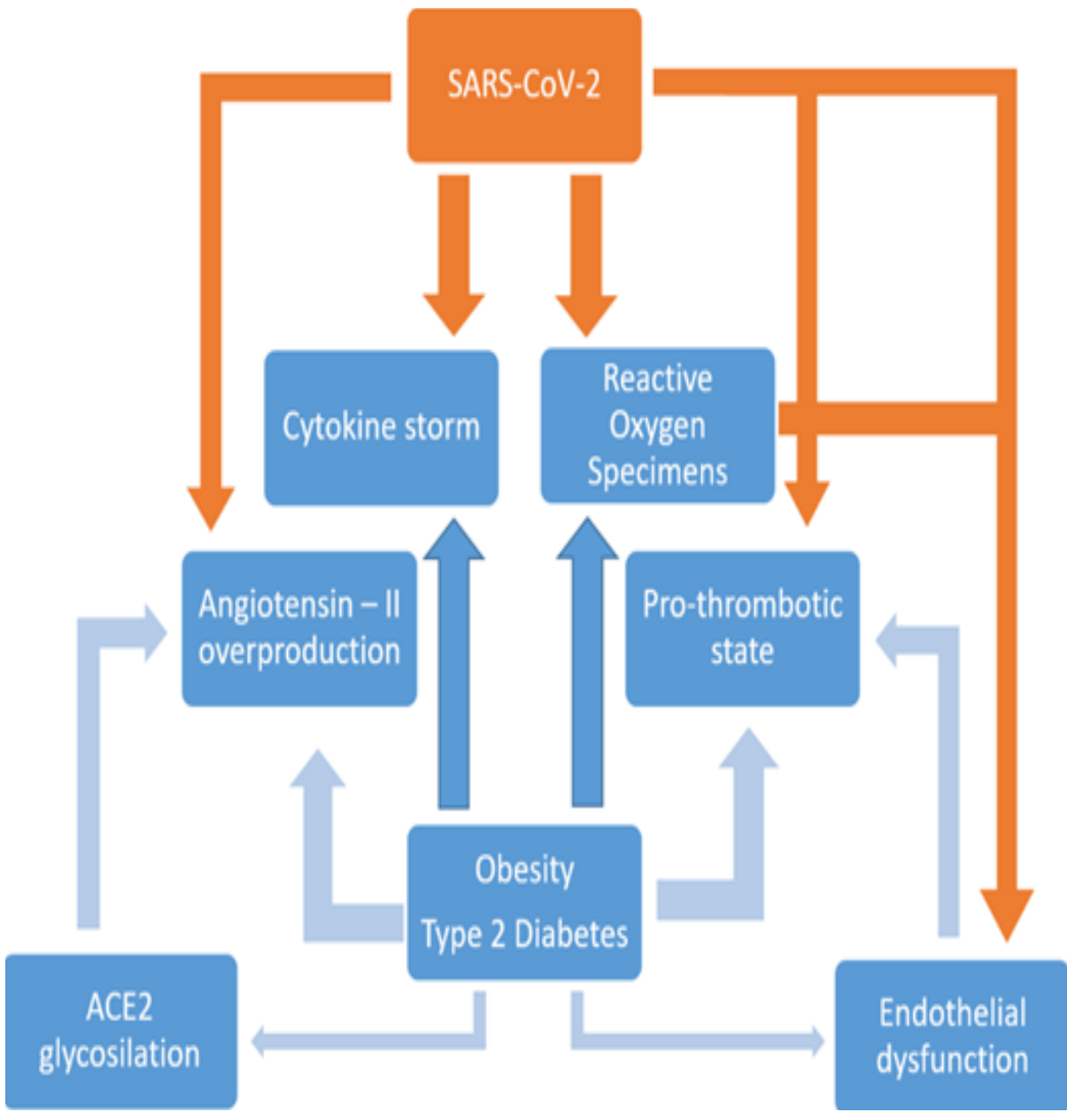

Figure I Plausible associations between metabolic diseases (obesity and type 2 diabetes mellitus) and SARS-CoV-2 infection, through feedback interaction and amplifying responses, determining severe cases. 


\section{ACE $2 \times$ COVID}

SARS-CoV-2 uses the ACE2 to infect cells, ${ }^{32}$ as it reduces the expression of ACE2, activation of the renin-angiotensin system (RAS) through ACE is amplified. Thus, the coexistence of COVID-19 and obesity, as well as T2DM (both conditions with RAS hyperactivation)can promote deleterious effects in different tissues, through the Angiotensin-1 receptor (AT1R) activation. ${ }^{33}$ ACE2, the binding site of SARS-CoV-2, is a critical component of the RAS counterregulatory pathway, which is one of the most important regulators of inflammation, blood pressure and fibrosis, and is determining in the pathophysiology of hypertension, cardiovascular disease, and chronic kidney disease. ${ }^{34}$ ACE2 converts Angiotensin II into Ang1-7, which acts in the Mas receptor to lower blood pressure and reduce fibrosis and inflammation. ${ }^{35}$ Ang 1-7 promotes vasodilation, reduces sympathetic tone, and increases nitric oxide production, being associated with a protective role in vascular diseases. ${ }^{36}$ Endocytosis of the SARS-CoV2-ACE2 complex, as well as virus-induced shedding of the ACE2 cell surface and ACE2 down regulation, all may contribute to decreased ACE2 expression and activity in infected cells. ${ }^{32}$ It may be a causal factor of the disease itself, due to an exaggerated activation of the
ACE/AngII/AT1R arm, leading to pulmonary injury, hematological alterations and hyper inflammatory state ${ }^{37}$ and a deleterious activation of the innate and adaptive immune response..$^{38}$ The down-regulation of ACE2 and consequent increased of Ang II, once this molecule is not converted into Ang1-7 results in increased pro-inflammatory cytokines, including IL-1, IL- 6 and TNF- $\alpha$, intensified by the activation of innate and adaptive immune response. ${ }^{39}$ Due to ACE/Ang II/AT1R axis activation and ACE2/Ang1-7/MasR axis down-regulation (that are already unbalanced in diabetic patients), the mortality and severity rates of COVID-19 may be higher. ${ }^{2}$ Implications of the ACE/ACE2 pathway on disease physiopathology is depicted on Figure 2. Initially, there was concern regarding the use of anti-hypertensive drugs that act in the RAS, as ACE inhibitors, as well as angiotensin receptor blockers, cause increase expression of ACE2, ${ }^{40}$ and so such drugs could facilitate viral infection. Recent data, however, shows that the use of drugs that act in the RAS, first choice as anti-hypertension therapy in diabetic patients, was not associated with worst prognosis. ${ }^{41}$ In fact, some cohorts demonstrated that its use was even associated with better survival, though data is controversial, and article has been retracted. ${ }^{42}$

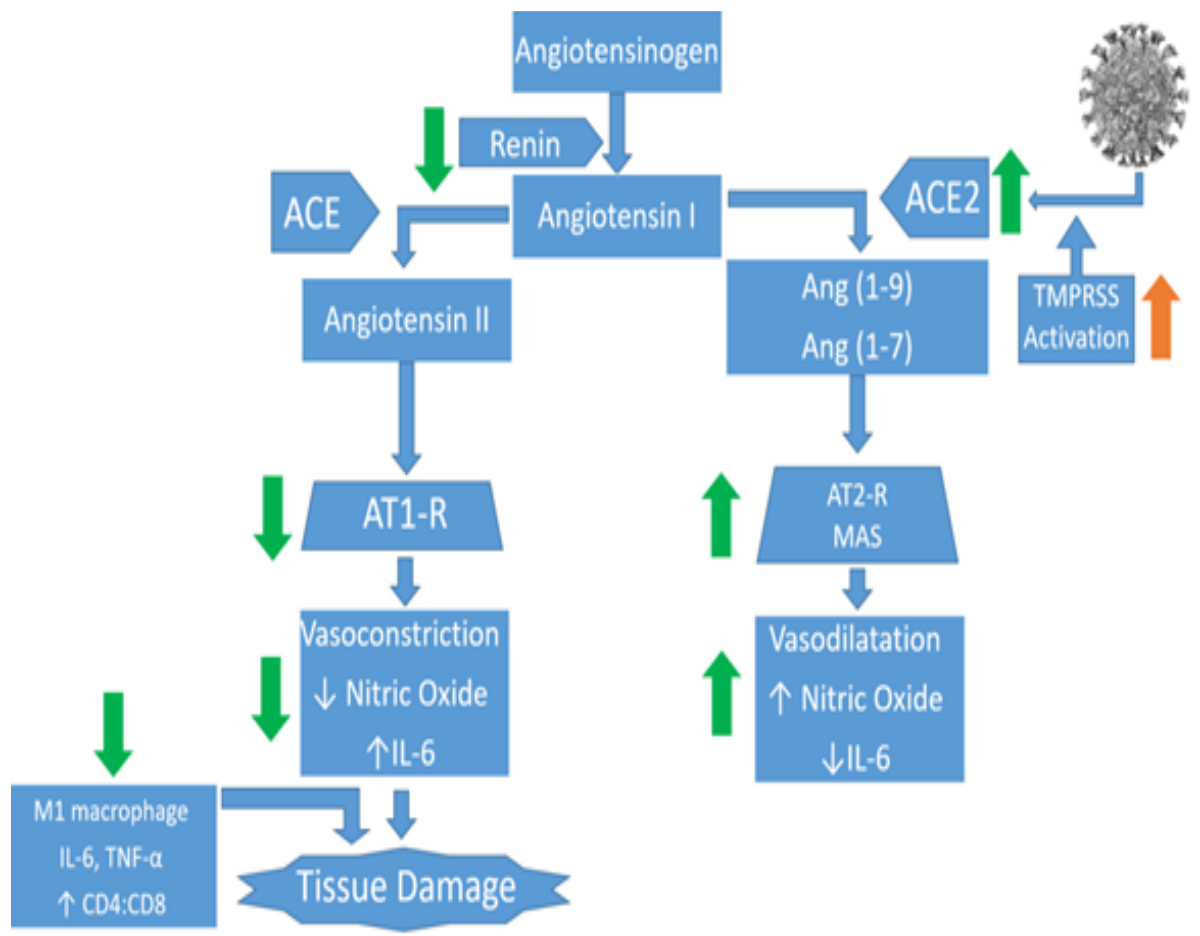

Figure 2 Plausible interactions between sexual steroids and viral invasion and immune response. Orange arrow indicates testosterone action; green arrows indicate estrogen action.

\section{Covid-I9 and vitamin d deficiency}

Vitamin D deficiency is a global unrecognized epidemic, afflicting both children and adults; although data is scarce, it is speculated that about one billion people around the world have low vitamin D levels, with some regional variations, especially due to solar exposure and skin tone from particular countries population. ${ }^{43}$ After emergence of COVID-19 in China and posterior proliferation all over the world, studies tried to associate the low levels of vitamin D seen in north European countries as a factor determining severity of the disease. A review showed the relation between latitude and mortality per million. ${ }^{44}$ This review plotted the mortality per million against latitude and it can be seen that all the countries that lie below 35 degree north have relatively low mortality; that latitude was chosen because it also happens to be the latitude in which sufficient levels of sunlight per day occur to retain adequate levels of vitamin D during the winter. Results pointed San Marino, Italy, Belgium and Spain, all above latitude 35 degrees north, as the countries with the highest number of cases, with a correlation between mortality and latitude of $\mathrm{r}=0.53$ and $\mathrm{P}<0.0001$ by Spearsman's rank; so it has been suggested the possible role of vitamin $\mathrm{D}$ in determining more severe outcomes in COVID-19.44 
Unfortunately, these studies showed major problems with confounding factors, such as age, ethnicity and obesity prevalence variations, as well as different detection and notification methods between the countries.

Preliminary data suggests that vitamin D participates in regulating, suppressing hyper-inflammatory, and cytokine response to various pathogens, including respiratory viruses, and thus could prevent cytokine storm; also, it has been reported that deficiency of vitamin D can increase thrombotic episodes with an elevation of D-dimer levels, which are frequently seen in severe COVID-19.45

However, a review analyzed the completed data of the UK Biobank participants, ${ }^{46}$ with over 348 thousand people, but only 449 had confirmed Covid-19 infection. Vitamin D was associated with COVID-19 infection univariably (OR $=0.99 ; 95 \%$ CI 0.99 $0.999 ; p=0.013)$ but not after adjustment for covariates $(\mathrm{OR}=1.00$; $95 \% \mathrm{CI}=0.998-1.01 ; p=0.208)$; ethnicity was associated with COVID-19 infection univariably (blacks versus whites $\mathrm{OR}=5.32$, $95 \% \mathrm{CI}=3.68-7.70, p$-value $<0.001)$, and the adjustment of $25(\mathrm{OH})$ $\mathrm{D}$ concentration made little difference to the magnitude of the association. Moreover, more robust resultsfailed toevidence that vitamin D supplementation in black people and Asians could prevent the serious Covid-19; the findings do not support a link between vitamin D levels and risk of Covid-19 infection, nor that vitamin D concentration may explain the ethnic variations in Covid-19 illness.

\section{Gender differences of severity and outcomes}

As the COVID-19 outbreak spread, differences between men and women began to emerge; though there is no significant difference on the incidence of cases, a clear disparity in severity has been continually reported, with men showing higher rates of severe cases and mortality, according to the Global Health 50/50 research initiative.$^{47}$ An European epidemiological study, analyzing confirmed COVID-19 cases from Italy, China, Spain, France, Germany, and Switzerland revealed that Intensive Care Unit cases and mortality was $50 \%$ to $400 \%$ higher amongst men. Interestingly, such difference was even more highlighted in younger patients, with ages up to 60 yearsold. ${ }^{4}$ Such dimorphism may rise from sexual steroids interferences on the viral cell invasion mechanisms. Estrogen has been associated with the differences between immune responses from men and women, as women present more effective $\mathrm{B}$ and $\mathrm{T}$ cell responses, with a preference of M2 macrophage response, higher CD4:CD8 ratios and lower IL-6 levels; ${ }^{48,49}$ this could be associated with a less intense cytokine storm during SARS-CoV-2 infection. Moreover, downregulation of AT1R by estrogens, and regulation of renin activity have been described, as well downregulation of ACE and simultaneous upregulation of ACE2, AT2R, and MAS expression levels, ${ }^{50}$ thus decreasing tissue damage.

Furthermore, testosterone may also play a role in this gender imbalance, as it increases cell-surface serine protease TMPRSS2, an activator of the Spike protein of the SARS-Cov-2, enabling its coupling with ACE-2, and then, viral internalization..$^{51}$ This could imply, then, that viral invasion in men is higher, as well as the deleterious hyperinflammatory response that leads to tissue damage. Figure 2 elicits sexual steroid interactions, leading to a higher inflammatory response in males, as well as ACE interaction.

A recent case-control study in Italy showed that men in androgen deprivation therapy (ADT) for prostate cancer had 4-fold lower odds of developing severe COVID-19 and death than those who were not taking ADT, though not adjusted for confounding factors. ${ }^{52}$ A trial to evaluate the use an estrogen patch has been registered in the US Clinical Trials Database.

\section{Conclusion}

There is a conundrum involving SARS-CoV-2 infection, host intrinsic risk factors, as well as immune response to infection, that is still due solving. However, as the pandemic continues afflicting humankind, better knowledge of such physiopathological mechanisms is acquired, and the search for a highly effective treatment is still the center of attention on scientific debates. In this review, using the available evidence, we seek to bring together in a single article the various mechanisms involving COVID-19 infection severity and endocrine aspects, as they may play an important role in disease course. The results of ongoing clinical trials are expected to resolve these issues.

\section{Declaration of conflicting interests}

The Authors declares that there is no conflict of interest.

\section{Acknowledgments}

None.

\section{References}

1. Zhu N, Zhang D, Wang W, et al. A Novel Coronavirus from Patients with Pneumonia in China, 2019. N Engl J Med. 2020;382(8):727-733.

2. Wu J, Zhang J, Sun X, et al. Influence of diabetes mellitus on the severity and fatality of SARS-CoV-2 (COVID-19) infection [published online ahead of print, 2020 Jun 4]. Diabetes ObesMetab. 2020;10.1111/ dom.14105.

3. Lighter J, Phillips M, Hochman S, et al. Obesity in Patients Younger Than 60 Years Is a Risk Factor for COVID-19 Hospital Admission. Clin Infect Dis. 2020;71(15):896-897.

4. Gebhard C, Regitz-Zagrosek V, Neuhauser HK, et al. Impact of sex and gender on COVID-19 outcomes in Europe. Biol Sex Differ. 2020;11(1):29.

5. Shi Y, Yu X, Zhao H, et al. Host susceptibility to severe COVID-19 and establishment of a host risk score: findings of 487 cases outside Wuhan. Crit Care. 2020;24(1):108.

6. Caussy C, Pattou F, Wallet F, et al. Prevalence of obesity among adult inpatients with COVID-19 in France. Lancet Diabetes Endocrinol. 2020;8(7):562-564

7. Melo LC, Silva MA, Calles AC. Obesity and lung function: a systematic review. Einstein (Sao Paulo). 2014;12(1):120-125.

8. Giannis D, Ziogas IA, Gianni P. Coagulation disorders in coronavirus infected patients: COVID-19, SARS-CoV-1, MERS-CoV and lessons from the past. J ClinVirol. 2020;127:104362.

9. Wichmann D, Sperhake JP, Lütgehetmann M, et al. Autopsy Findings and Venous Thromboembolism in Patients With COVID-19 [published online ahead of print, 2020 May 6]. Ann Intern Med. 2020;M20-2003.

10. Guan WJ, Ni ZY, Hu Y, et al. Clinical Characteristics of Coronavirus Disease 2019 in China. N Engl J Med. 2020;382(18):1708-1720.

11. Chiappetta S, Schaack HM, Wölnerhannsen B, et al. The Impact of Obesity and Metabolic Surgery on Chronic Inflammation [published correction appears in Obes Surg. 2018 Jun 27;:]. ObesSurg. 2018;28(10):3028-3040.

12. Frydrych LM, Bian G, O'Lone DE, et al. Obesity and type 2 diabetes mellitus drive immune dysfunction, infection development, and sepsis mortality. J Leukoc Biol. 2018;104(3):525-534. 
13. Chiappetta S, Jamadar P, Stier C, et al. The role of C-reactive protein after surgery for obesity and metabolic disorders. Surg ObesR elatDis. 2020;16(1):99-108.

14. Blind E, Janssen H, Dunder K, et al. The European Medicines Agency's approval of new medicines for type 2 diabetes. Diabetes ObesMetab. 2018;20(9):2059-2063.

15. Klekotka RB, Mizgała E, Król W. The etiology of lower respiratory tract infections in people with diabetes. PneumonolAlergol Pol. 2015;83(5):401-408.

16. Huang I, Lim MA, Pranata R. Diabetes mellitus is associated with increased mortality and severity of disease in COVID-19 pneumonia - A systematic review, meta-analysis, and meta-regression. Diabetes MetabSyndr. 2020;14(4):395-403.

17. Knapp S. Diabetes and infection: is there a link?-A minireview. Gerontology. 2013;59(2):99-104.

18. González Plaza JJ, Hulak N, Akilzhanova A, et al. Role of metabolism during viral infections, and crosstalk with the innate immune system. IntractableRareDis Res. 2016;5(2):90-96.

19. Guo W, Li M, Dong Y, et al. Diabetes is a risk factor for the progression and prognosis of COVID-19 [published online ahead of print, 2020 Mar 31]. Diabetes Metab Res Rev. 2020;e3319.

20. Toniolo A, Cassani G, Puggioni A, et al. The diabetes pandemic and associated infections: suggestions for clinical microbiology. Rev Med Microbiol. 2019;30(1):1-17.

21. Moore JB, June $\mathrm{CH}$. Cytokine release syndrome in severe COVID-19. Science. 2020;368(6490):473-474.

22. Cameron AR, Morrison VL, Levin D, et al. Anti-Inflammatory Effects of Metformin Irrespective of Diabetes Status. Circ Res. 2016;119(5):652-665.

23. Bramante $\mathrm{C}$, Ingraham $\mathrm{N}$, Murray $\mathrm{T}$, et al. Observational Study of Metformin and Risk of Mortality in Patients Hospitalized with Covid-19. Preprint. medRxiv. 2020;2020.06.19.20135095.

24. Luo P, Qiu L, Liu Y, et al. Metformin Treatment Was Associated with Decreased Mortality in COVID-19 Patients with Diabetes in Retrospective Analysis. Am J Trop Med Hyg. 2020;103(1):69-72.

25. Singh AK, Singh R. Does poor glucose control increase the severity and mortality in patients with diabetes and COVID-19? [published online ahead of print, 2020 May 27]. Diabetes MetabSyndr. 2020;14(5):725-727.

26. Bode B, Garrett V, Messler J, et al. Glycemic Characteristics and Clinical Outcomes of COVID-19 Patients Hospitalized in the United States [published correction appears in J Diabetes Sci Technol. 2020 Jun 10;:1932296820932678]. J Diabetes SciTechnol. 2020;14(4):813-821.

27. Ceriello A, De Nigris V, Prattichizzo F. Why is hyperglycaemia worsening COVID-19 and its prognosis? [published online ahead of print, 2020 May 28]. Diabetes ObesMetab. 2020;10.1111/dom.14098.

28. Satoh-Asahara N, Sasaki Y, Wada H, et al. A dipeptidyl peptidase-4 inhibitor, sitagliptin, exerts anti-inflammatory effects in type 2 diabetic patients. Metabolism. 2013;62(3):347-351.

29. Matsubara J, Sugiyama S, Akiyama E, et al. Dipeptidyl peptidase-4 inhibitor, sitagliptin, improves endothelial dysfunction in association with its anti-inflammatory effects in patients with coronary artery disease and uncontrolled diabetes. Circ J. 2013;77(5):1337-1344.

30. Raj VS, Mou H, Smits SL, et al. Dipeptidyl peptidase 4 is a functional receptor for the emerging human coronavirus-EMC. Nature. 2013;495(7440):251-254.

31. Fadini GP, Morieri ML, Longato E, et al. Exposure to dipeptidylpeptidase-4 inhibitors and COVID-19 among people with type 2 diabetes: A case-control study [published online ahead of print, 2020 May 28]. Diabetes ObesMetab. 2020;10.1111/dom.14097.

32. Hoffmann M, Kleine-Weber H, Schroeder S, et al. SARS-CoV-2 Cell Entry Depends on ACE2 and TMPRSS2 and Is Blocked by a Clinically Proven Protease Inhibitor. Cell. 2020;181(2):271-280.e8.
33. Zhu L, She ZG, Cheng X, et al. Association of Blood Glucose Control and Outcomes in Patients with COVID-19 and Pre-existing Type 2 Diabetes. Cell Metab. 2020;31(6):1068-1077.e3.

34. South AM, Brady TM, Flynn JT. ACE2 (Angiotensin-Converting Enzyme 2), COVID-19, and ACE Inhibitor and Ang II (Angiotensin II) Receptor Blocker Use During the Pandemic: The Pediatric Perspective. Hypertension. 2020;76(1):16-22.

35. South AM, Shaltout HA, Washburn LK, et al. Fetal programming and the angiotensin-(1-7) axis: a review of the experimental and clinical data. ClinSci (Lond). 2019;133(1):55-74.

36. Sampaio WO, Souza dos Santos RA, Faria-Silva R, et al. Angiotensin(1-7) through receptor Mas mediates endothelial nitric oxide synthase activation via Akt-dependent pathways. Hypertension. 2007;49(1):185-192.

37. Lanza K, Perez LG, Costa LB, et al. Covid-19: the renin-angiotensin system imbalance hypothesis. ClinSci (Lond). 2020;134(11):1259-1264.

38. Imai Y, Kuba K, Rao S, et al. Angiotensin-converting enzyme 2 protects from severe acute lung failure. Nature. 2005;436(7047):112-116.

39. Tanaka T, Narazaki M, Masuda K, et al. Regulation of IL-6 in Immunity and Diseases. Adv ExpMed Biol. 2016;941:79-88.

40. Soler MJ, Barrios C, Oliva R, Batlle D. Pharmacologic modulation of ACE2 expression. Curr Hypertens Rep. 2008;10(5):410-414.

41. Fernández-Ruiz I. RAAS inhibitors do not increase the risk of COVID-19. Nat RevCardiol. 2020;17(7):383.

42. Mehra MR, Desai SS, Kuy S, et al. Cardiovascular Disease, Drug Therapy, and Mortality in Covid-19 [retracted in: N Engl J Med. 2020 Jun 4;:]. N Engl J Med. 2020;382(25):e102.

43. Palacios C, Gonzalez L. Is vitamin D deficiency a major global public health problem?. J Steroid Biochem Mol Biol. 2014;144 Pt A:138-145.

44. Rhodes JM, Subramanian S, Laird E, et al. Editorial: low population mortality from COVID-19 in countries south of latitude 35 degrees North supports vitamin D as a factor determining severity. AlimentPharmacolTher. 2020;51(12):1434-1437.

45. Fisher SA, Rahimzadeh M, Brierley C, et al. The role of vitamin D in increasing circulating $\mathrm{T}$ regulatory cell numbers and modulating $\mathrm{T}$ regulatory cell phenotypes in patients with inflammatory disease or in healthy volunteers: A systematic review. PLoSOne. 2019;14(9):e0222313.

46. Hastie CE, Mackay DF, Ho F, et al. Vitamin D concentrations and COVID-19 infection in UK Biobank [published correction appears in Diabetes MetabSyndr. 2020 Jul 23;14(5):1315-1316]. Diabetes MetabSyndr. 2020;14(4):561-565.

47. UK Research and innovation 2020, Sex, gender and Covid-19, Global health 5050 .

48. Wikby A, Månsson IA, Johansson $\mathrm{B}$, et al. The immune risk profile is associated with age and gender: findings from three Swedish population studies of individuals 20-100 years of age. Biogerontology. 2008;9(5):299-308

49. Leng SX, Margolick JB. Aging, sex, inflammation, frailty, and CMV and HIV infections. CellImmunol. 2020;348:104024.

50. Regitz-Zagrosek V, Seeland U. Sex and gender differences in myocardial hypertrophy and heart failure. Wien MedWochenschr. 2011;161(5-6):109-116.

51. Tomlins SA, Rhodes DR, Perner S, et al. Recurrent fusion of TMPRSS2 and ETS transcription factor genes in prostate cancer. Science. 2005;310(5748):644-648.

52. Montopoli M, Zumerle S, Vettor R, et al. Androgen-deprivation therapies for prostate cancer and risk of infection by SARS-CoV-2: a populationbased study (N = 4532). Ann Oncol. 2020;31(8):1040-1045. 